\title{
Pharmacovigilance: A Step Towards Patient Safety
}

\author{
Dr. Pramod Kumar Manjhi \\ Head, Department of Pharmacology, All India Institute of Medical Sciences, Phulwarisharif, India. \\ Email:drpramodkumar@aiimspatna.org
}

ISI Impact Factor

(2019-20): 1.628

IC Value (2019): 90.81

SJIF (2020) $=7.893$

(c) (i) (3)

Copyright@IJCRR

The advent of pharmacovigilance occurred on a global scale with the infamous Thalidomide disaster (1961- 1962). The drug was used to treat sleeplessness, nausea and morning sickness in the post-world war II era. According to the laws existing at that period, only a little research regarding the safety of drugs was carried out in animal experiments, still, it was marketed as safe to even to pregnant women. It is estimated that around 10000 fetuses were affected by this leading to worldwide consensus over the need for pharmacovigilance. ${ }^{1}$

Pharmacovigilance is defined by $\mathrm{WHO}$ as the science and activities relating to the detection, assessment, understanding and prevention of adverse effects or any other medicine/ vaccine-related problem. ${ }^{2}$

The Pharmacovigilance Programme of India (PvPI) inducted in 2010 under CDSCO and MoHFW- Govt. of India, is the body that spearheads the pharmacovigilance activities in India with its National Coordinating Centre (NCC) situated in Ghaziabad Uttar Pradesh. ${ }^{3}$ The data is collected from all over the country via AMC (Adverse drug reactions Monitoring Centre) to be compiled and analyzed. The objectives that PVPI-NCC wishes to satisfy include: 1) Monitoring ADR 2) Creating awareness among Health care professionals (HCP) 3)To generate data on the safety of medicine 4) To help CDSCO form regulatory decisions.

The reporting of any adverse event/ adverse drug reaction can be done by either an HCP or a consumer using any of the following methods: 1) Mobile application 2) AMC 3) ADR form 4) toll-free helpline. All the data collected, is reported along with the causality assessment to the NCC -PVPI. Here the data is analyzed and, causality assessment is rechecked and new signals are detected. They can also send out advisory or lookout notices to prevent further Adverse drug events. The data flow and analysis is greatly helped by the development of Artificial Intelligence. The data received by AMC reach NCC via VigiFlow software which is finally channelised to Uppsala Monitoring Centre (Sweden). VigiBase is the WHO global database of individual case safety reports. VigiLyze is an online resource that provides a quick and clear overview of VigiBase, vigiGrade is a measure of report quality, vigiMatch an algorithm for automated duplicate detection whereas vigiRank is a statistical signal detection method for Pharmacovigilance. ${ }^{4}$ To prevent ADR use drugs only when required, take a detailed drug history and select rational drugs and avoid unnecessary drug combinations, irrational use and abrupt withdrawal.

The major benefits that are seen by pharmacovigilance include 1) Safety assessment of drug therapy 2) to release drug safety information on a timely basis 3 ) Assess the economic impact of Adverse drug events 4) Regulatory action on drugs on basis of ADR reporting. ${ }^{5}$

\section{CONCLUSION}

There is an urgent need to increase awareness to strengthen pharmacovigilance in the country and around the globe. It requires active participation from all chains of the health care sector, starting from the patient to identify his adverse event, then the HCP to report it correctly followed by the PvPI and WHO to coordinate, analyze and come up with the required policy to implement and direct the government to ensure patient safety.

\section{REFERENCES}

1. Therapeutic disasters that hastened safety testing of new drugs - PubMed [Internet]. [cited 2021 Oct 8]. Available from: https:// pubmed.ncbi.nlm.nih.gov/28318023/

2. Regulation and Prequalification [Internet]. [cited 2021 Oct 8]. Available from: https:/www.who.int/teams/regulation-prequalification/pharmacovigilance

3. Kalaiselvan V, Srivastava S, Singh A, Gupta SK. Pharmacovigilance in India: Present Scenario and Future Challenges. Drug Saf. 2019 Mar;42(3):339-46.

4. vigi_tools_and_methods.pdf [Internet]. [cited 2021 Oct 8]. Available from: https://www.who-umc.org/media/3086/vigi tools_and_methods.pdf

5. Beninger $\bar{P}$. Pharmacovigilance: An Overview. Clin Ther. 2018 Dec;40(12):1991-2004. 